\title{
La phagothérapie : cauchemar pour la bactérie et rêve pour le médecin?
}

\author{
Laurent Debarbieux ${ }^{1}$, Emilie Saussereau ${ }^{1}$ et Damien Maura ${ }^{2,3,4}$ \\ 1 Institut Pasteur, Département de Microbiologie, 25 Rue du Dr Roux, 75724 Paris Cedex 15, France \\ 2 Department of Surgery, Harvard Medical School and Massachusetts General Hospital, Boston, \\ 02114 Massachusetts, USA \\ 3 Department of Microbiology and Immunobiology, Harvard Medical School, Boston, 02114 Massachusetts, USA \\ 4 Shriners Hospitals for Children-Boston, Boston, 02114 Massachusetts, USA
}

Auteur correspondant : Laurent Debarbieux, laurent.debarbieux@pasteur.fr

Reçu le 5 juin 2013

\begin{abstract}
Résumé - Découverts au début du $\mathrm{XX}^{\mathrm{e}}$ siècle, les bactériophages ont rapidement été utilisés pour traiter des infections bactériennes, devenant ainsi les premiers antibactériens spécifiques à être employés en médecine. Cependant, l'arrivée des antibiotiques a brutalement réduit leur utilisation, les confinant au rayon « boîte à outils » du chercheur. Celui-ci en fît bon usage permettant de dévoiler une très grande partie des secrets moléculaires de la cellule. Aujourd'hui, face à la menace que représentent les pathogènes bactériens résistants aux antibiotiques, les bactériophages tentent de faire leur retour dans le domaine médical. Bien que la connaissance de ces virus bactériens ait considérablement progressé après presque un siècle d'études, leur utilisation thérapeutique est loin d'être maîtrisée. Cet article relate, à l'aide de deux exemples récents de l'utilisation expérimentale des bactériophages, les espoirs mais aussi les challenges qui entourent la mise en place de la phagothérapie appliquée à l'Homme.
\end{abstract}

Mots clés : Bactériophages thérapeutiques / infections bactériennes / résistance aux antibiotiques / pneumonie / diarrhée

Abstract - Phagotherapy: a nightmare for bacteria, a dream for physicians?

Bacteriophages were discovered in the early 20th century and rapidly used to treat bacterial infections in humans. As the first specific antibacterial agents, they were used worldwide until antibiotics ramped up. Thereafter, rapidly forgotten, they became the favorite toolbox for researchers that used them to elucidate some of the most fundamental aspects of the cellular life at the molecular level. Today, facing the threat of antibiotic resistant bacteria, bacteriophages are being reconsidered for their use in medicine. During the past century, knowledge on bacteriophages has improved considerably, nevertheless phage therapy is still in its infancy. Taking two examples of recently published experimental phage therapy results, this article summarizes the hopes but also the challenges that surround the future development of human phage therapy.

Key words: Phage therapy / bacterial infections / antibiotics resistance / pneumonia / diarrhea

\section{Introduction}

La phagothérapie est associée au nom de Félix d'Herelle, un chercheur autodidacte du début du
XXe siècle (Summers, 1999; Dublanchet \& Fruciano, 2008). Travaillant alors à l'Institut Pasteur, il a décrit en 1917 un «principe » qui détruisait les bactéries et qu'il nomma bactériophages, qui mot à mot signifie 
《 mangeur de bâtons », les bâtons faisant référence à la forme de certaines bactéries. Les bactéries détruites étant des agents pathogènes pour l'Homme, il en conclut que ces bactériophages pouvaient permettre de soigner des patients atteints d'infections bactériennes. Ainsi, ses toutes premières tentatives de phagothérapie chez des enfants à l'Hôpital NeckerEnfants Malades de Paris ont été effectuées en 1919, après qu'il eut préalablement absorbé et fait absorber la préparation de bactériophages à son entourage pour en vérifier l'innocuité. Cinq enfants atteints de dysenterie bacillaire furent ainsi traités avec succès. Dès lors, ce traitement, qui à l'époque fût le premier traitement antibactérien spécifique, s'est développé à travers le monde. Dès 1921, le « phénomène de d'Herelle » fut introduit aux EtatsUnis par Gratia, qui publia ensuite les résultats du traitement d'une cinquantaine de patients atteints d'infections à Staphylococcus (Gratia, 1921, 1922). Puis, nombreux furent ceux qui s'appliquèrent à traiter diverses pathologies : fièvres typhoïdes, paratyphoïdes, infections urinaires, suppurations staphylococciques et streptococciques chirurgicales ou cutanées etc. En 1924, la phagothérapie était devenue le traitement de routine pour la dysenterie bacillaire au Brésil (Da Costa Cruz, 1924). En 1926, Félix d'Herelle écrivit un livre qui dressait l'inventaire des possibilités thérapeutiques décrites à cette époque. Les références très nombreuses (près de 700) montrent à quel point cette thérapie avait pris de l'importance (d'Herelle, 1926). Les travaux de d'Herelle sur le traitement de la peste bubonique par les bactériophages accentuèrent encore davantage la renommée mondiale de la phagothérapie (d'Herelle, 1925). S'en suivit une utilisation massive des bactériophages pour traiter la peste et le choléra en Inde durant une dizaine d'années. À la vue de ce succès grandissant, plusieurs entreprises pharmaceutiques importantes telles que Parke-Davis, Eli Lilly ou Robert \& Carrière commercialisèrent des préparations de bactériophages à usage thérapeutique. À partir de 1928, d'Herelle installa son laboratoire au sein de l'entreprise Robert \& Carrière en France et y développa des préparations de bactériophages (bacté-intesti-phage, bacté-coli-phage, bacté-rhino-phage, bacté-pyophage et bacté-staphyphage) qui firent partie des dix meilleures ventes des médicaments de l'entreprise Robert \& Carrière pendant les années 1930, et dont certaines étaient encore référencées dans le Vidal dans les années 1970 (Dublanchet \& Fruciano, 2008).

À côté des succès rapportés, il y eut aussi des échecs et une étude commandée par l'Association Américaine de Médecine menée par Eaton et Bayne-Jones en 1934 conclut que, mis à part l'utilisation des bactériophages contre les infections à staphylocoques, les résultats n'étaient globalement pas convaincants. Ces auteurs signalaient également des problèmes de stabilité des préparations ainsi que des compositions variables et mal définies (il s'agissait généralement de cocktails de plusieurs bactériophages) (Dublanchet \& Fruciano, 2008). Il faut rappeler qu'à cette époque la nature même des bactériophages était encore sujette à débats. En effet, bien que d'Herelle ait dans sa publication originale de 1917 décrit les expériences qui permettaient de définir les bactériophages comme des virus, il n'a franchi le pas que quelques années plus tard. En effet, la communauté scientifique a longtemps douté de la nature virale de ces bactériophages. Il faut reconnaître qu'à l'époque, les virus n'avaient été décrits que pour le domaine des animaux et des végétaux, et l'étude des bactéries était encore assez récente. Finalement, la véritable nature virale des bactériophages fut démontrée à l'aube de la seconde guerre mondiale grâce au microscope électronique (Luria et al., 1943). Aujourd'hui nous savons que les virus sont ubiquitaires, capables d'infecter toutes les cellules, qu'elles appartiennent aux domaines des Archaea, Bactéries ou Eukaryotes (Prangishvili et al., 2006).

L'arrivée de la pénicilline, découverte par Alexander Flemming, contribua fortement au déclin de la phagothérapie (Summers, 2012). Plus facile d'emploi, plus stable et d'une efficacité d'emblée miraculeuse sur certaines infections, l'antibiothérapie confina dès la fin de la seconde guerre mondiale l'utilisation médicale des bactériophages à une place anecdotique. Entre les années 1970 et 1990 apparurent sur le marché une trentaine de nouvelles molécules antibiotiques. Les infections bactériennes, sinon définitivement vaincues, semblaient être maîtrisées (Dublanchet \& Fruciano, 2008). Bien que très tôt décrite par Flemming lui-même, la résistance des bactéries à la pénicilline n'était que le début d'un phénomène naturel dont les conséquences ont été dramatiquement sous-estimées. Après une période florissante, l'investissement des entreprises pharmaceutiques dans la recherche d'antibiotiques ainsi que l'augmentation des coûts associés à l'obtention d'une autorisation de mise sur le marché a conduit à une forte réduction du nombre de nouveaux antibiotiques commercialisés depuis le début des années 1990. Parallèlement, on a pu observer sur la même période une forte augmentation de l'incidence de bactéries résistantes aux antibiotiques, tels que les Staphylocoques résistants à la Méthicilline (MRSA) ou les Entérocoques résistants à la Vancomycine (VRE) (figure 1) (Taubes, 2008). Parmi les nouvelles molécules antibiotiques en développement, rares sont celles qui représentent de nouvelles classes, la plupart étant des modifications chimiques et structurales de celles déjà existantes (Spellberg et al., 2004). Face à cette impasse qui se profile, plusieurs stratégies 
A

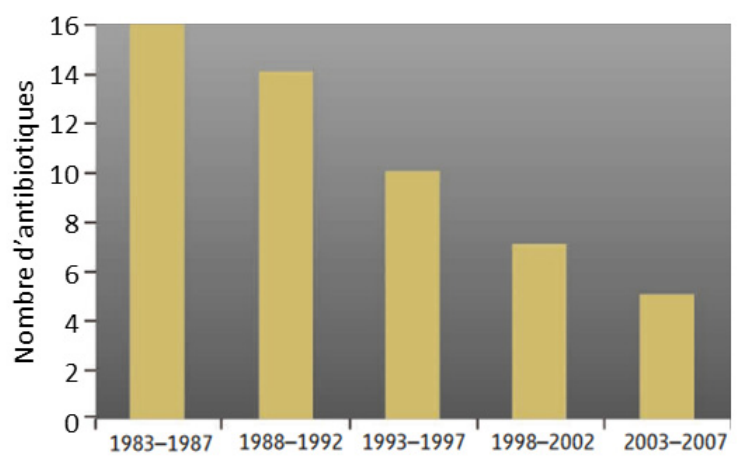

B

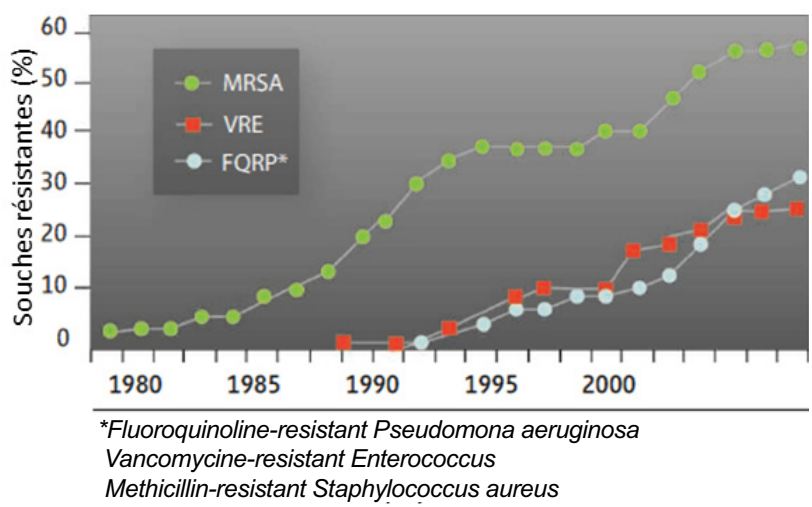

Fig. 1. Nombre de nouveaux antibiotiques approuvés par la Federal Food and Drug Administration (A). Évolution du pourcentage des souches résistantes aux antibiotiques au cours du temps (B) (d'après Taubes, 2008).

ont été envisagées, dont le renouveau de la phagothérapie. Celui-ci est d'autre part encouragé par la redécouverte que cette thérapie a été utilisée depuis les années 1920 dans certains pays de l'Europe de l'Est et est encore utilisée de nos jours, bien que moins fréquemment que par le passé (Kutateladze \& Adamia, 2010; Miedzybrodzki et al., 2012). Enfin, à partir des années 1940, les connaissances fondamentales sur les bactériophages n'ont cessé de croître, permettant l'essor d'une discipline à part entière, la biologie moléculaire (Sulakvelidze et al., 2001; Maura \& Debarbieux, 2011; Henry \& Debarbieux, 2012).

\section{Qu'est-ce qu'un bactériophage?}

Les bactériophages sont des virus n'infectant que les bactéries. Celles-ci étant faciles à manipuler au laboratoire, elles se sont rapidement imposées au début du siècle dernier comme modèles d'études pour comprendre les mécanismes sur lesquels repose la vie cellulaire. De manière concomitante, les bactériophages sont devenus des outils essentiels à cette quête. En 1943, Luria \& Delbrück démontrèrent avec leur test de fluctuation utilisant le bactériophage T1 que les mutations génétiques surviennent de manière aléatoire et sont ensuite sélectionnées, mais ne sont pas induites par la pression de sélection (Luria \& Delbruck, 1943). En 1952, Hershey et Chase confirmèrent que l'ADN était le support de l'information génétique des virus. À l'aide de bactériophages radio-marqués (avec du phosphate pour l'ADN et du soufre pour les protéines), ils observèrent que le phosphate radio-marqué était retrouvé à l'intérieur des cellules bactériennes, tandis que le soufre radiomarqué était, lui, retrouvé dans les particules virales restées accrochées à l'extérieur de la paroi de la bactérie. Ceci leur indiqua que l'ADN des bactériophages pénétrait dans les cellules bactériennes et était le matériel porteur de l'information génétique héréditaire. La même année, Luria et Human mirent en évidence l'existence d'enzymes de restriction produites par Escherichia coli lors de l'infection par le groupe de bactériophages T1-T7, enzymes qui perturbent le déroulement complet du cycle viral (Luria \& Human, 1952). En 1961, Crick et al. décryptèrent le code génétique et sa structure en triplets de nucléotides (codons) grâce à des mutations induites par la proflavine dans le gène $r I I B$ du bactériophage T4 (Crick et al., 1961). La même année, Brenner mit en évidence l'ARN messager comme intermédiaire entre l'ADN et les ribosomes, grâce à la synthèse protéique induite lors d'une infection de $E$. coli par le bactériophage T4 (Brenner et al., 1961). De nombreuses autres découvertes fondamentales l'ont été via l'utilisation de bactériophages comme outils, notamment la découverte du concept d'opéron, la régulation génétique et le mécanisme de lysogénie grâce en particulier à l'étude du bactériophage lambda. Enfin, ces dernières années, un nombre important d'applications biotechnologiques ont été développées à partir des bactériophages, dont la technique de «phage display » est sans doute la plus connue, et encore aujourd'hui de nouvelles applications se font jour dans le domaine des nanotechnologies (Dang et al., 2011; Henry \& Debarbieux, 2012).

Cependant un tel succès ne repose que sur la connaissance approfondie d'un nombre très limité de bactériophages en comparaison de la quantité de ces virus présents sur Terre. En effet, les premières études sur l'abondance des bactériophages dans l'environnement, réalisées grâce à la microscopie à épifluorescence, ont suggéré qu'il existait dix fois plus de particules virales que de cellules procaryotes en 


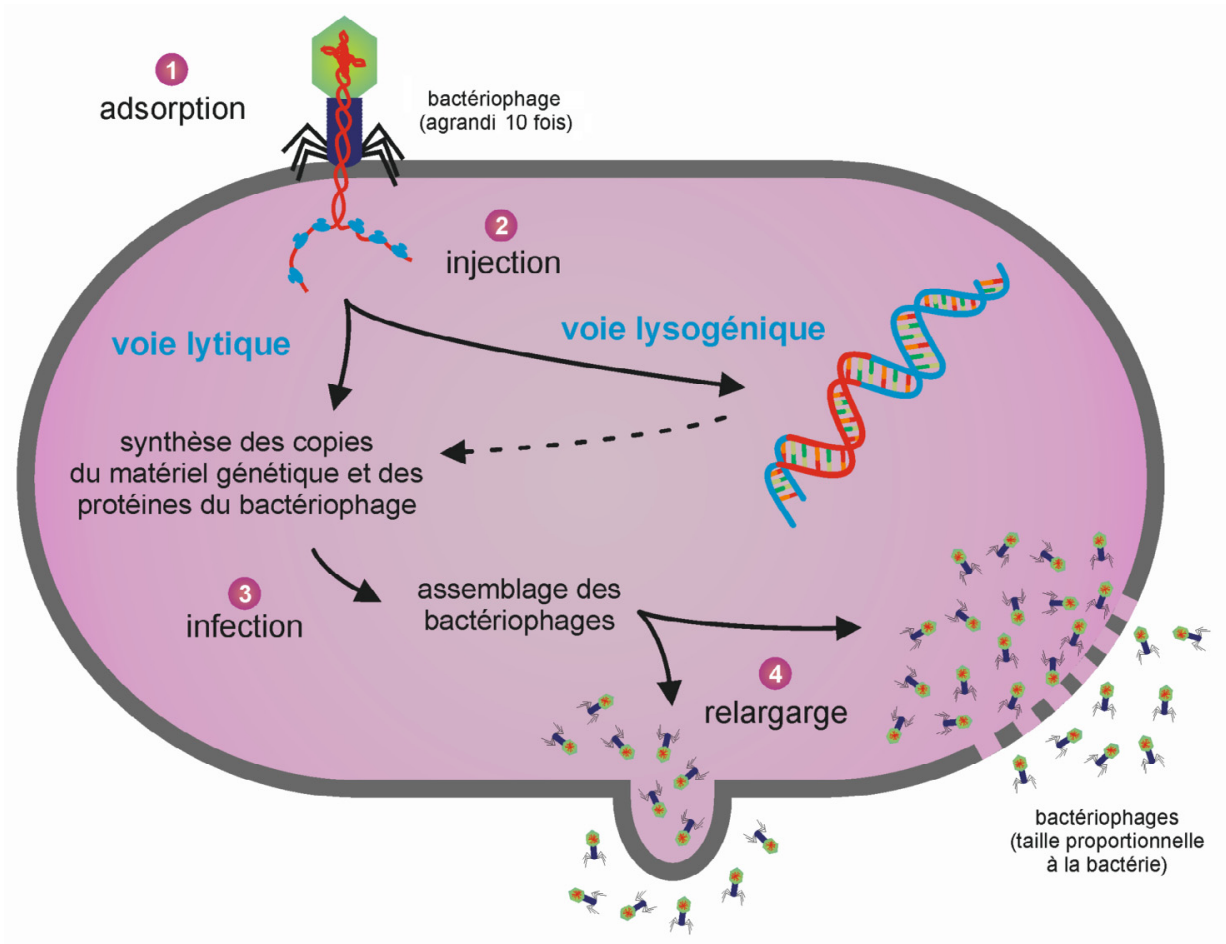

Fig. 2. Représentation schématique des principales étapes du cycle infectieux des bactériophages (modifiée d'après Maura \& Debarbieux, 2011). L'ovale violet représente la bactérie.

milieu aquatique, terrestre et sédimentaire (Breitbart \& Rohwer, 2005). Étant donné le nombre de procaryotes dans ces environnements, on estime à environ $10^{31}$ le nombre de particules virales à la surface de la Terre. Puisque la stabilité de ces virus est affectée par un grand nombre de paramètres environnementaux (lumière UV, température, $\mathrm{pH}, \ldots$...) leur maintien à un taux si élevé nécessite un renouvellement dynamique de leurs populations impliquant une infection massive de nombreux hôtes bactériens.

Ainsi, on estime que 20 à $50 \%$ de la mortalité bactérienne à l'échelle de la planète est due aux bactériophages, ce qui les place au même niveau que les autres grands prédateurs bactériens : les protistes unicellulaires. Ces infections relâchent de grandes quantités de composants cellulaires, augmentant ainsi le pool de matière organique particulaire et dissoute impliqué dans les cycles biogéochimiques (Wommack \& Colwell, 2000). Toutefois, le renouvellement de ces populations virales ne conduit pas à une diminution globale de la biomasse bactérienne. Des variations locales ont été observées, indiquant que les populations de bactéries et de bactériophages coexistent à travers une évolution dynamique (Faruque et al., 2005). Ainsi, la « course aux armements » entre bactériophages et bactéries est une force motrice majeure de l'évolution de ces deux types d'organismes, basée sur le développement de résistance aux bactériophages par les bactéries, et sur les moyens que ces virus utilisent pour les contourner (Labrie et al., 2010). Bien que de nombreuses études concernant l'importance écologique des bactériophages dans des environnements aquatiques, sédimentaires ou terrestres aient été entreprises, peu de choses sont connues en revanche sur l'importance écologique des bactériophages dans les environnements associés aux mammifères et particulièrement la flore microbienne du tractus gastro-intestinal (Clokie et al., 2011). Le rôle éventuel des bactériophages dans des pathologies intestinales a récemment été suggéré, mais de nombreuses questions restent en suspens (Lepage et al., 2008). Par exemple, quelle est l'importance écologique du transfert de gènes dans l'intestin via les bactériophages (Duerkop et al., 2012)? Quelles sont les raisons expliquant la dominance des bactériophages tempérés dans le microbiote intestinal, contrairement aux environnements aquatiques ou terrestres où prédominent les bactériophages virulents (Reyes et al., 2010) ? Deux très récents travaux illustrent les dernières avancées dans ce domaine (Modi et al., 2013; Minot et al., 2013).

Les bactériophages présentent plusieurs cycles infectieux différents, chacun avec ses propres caractéristiques (figure 2). Cependant, ils démarrent tous par la même étape : le virus s'adsorbe à la surface de la cellule bactérienne. Puis, le matériel 
génétique est injecté dans le cytoplasme bactérien par des mécanismes différents. Ensuite, plusieurs voies sont possibles.

- Les bactériophages à cycle lytique détournent rapidement les machineries de réplication, transcription et traduction de l'hôte à leur profit pour la production de virions. Ils transforment ainsi la cellule infectée en véritable usine à virus, qui sont dispersés à la fin du cycle dans le milieu extérieur via la lyse de la cellule infectée.

- Les bactériophages à cycle lysogénique se comportent souvent comme des virus à cycle lytique, mais parfois l'ADN du bactériophage est intégré dans le chromosome bactérien. Il persiste ainsi sous forme de prophage durant plusieurs générations à l'état « dormant » au sein de la bactérie, jusqu'à ce qu'un signal de stress provenant du milieu extérieur conduise à l'excision du prophage et au redémarrage d'un cycle lytique classique.

- Certains bactériophages peuvent présenter une étape pseudolysogénique dans leur cycle infectieux. Bien que ce concept soit encore imparfaitement défini, il se concrétise par le fait que le matériel génétique viral ne s'intègre pas dans le chromosome mais n'enclenche pas de cycle lytique non plus, jusqu'à ce qu'une condition favorable au démarrage d'un cycle lytique ou lysogénique apparaisse. On peut alors assimiler le génome du bactériophage à un plasmide (Clokie et al., 2011).

- Enfin, d'autres bactériophages possèdent un cycle chronique proche du cycle lytique mais qui diffère de celui-ci à l'étape de relargage des virions dans le milieu extérieur. Les virions produits lors d'un cycle chronique sont excrétés à travers la membrane sans que la bactérie ne soit lysée.

\section{La phagothérapie expérimentale}

Depuis un peu plus de vingt ans, plusieurs publications ont apporté des éléments tangibles quant au potentiel de la phagothérapie à l'aide de modèles animaux pour le traitement d'infections expérimentales variées (cutanées, intestinales, pulmonaires et de septicémie) (Maura \& Debarbieux, 2011; Saussereau \& Debarbieux, 2012). Nous aborderons en détail deux exemples actuellement à l'étude au laboratoire et qui permettent d'illustrer deux situations médicales différentes : les infections pulmonaires et les infections intestinales.

\section{Infections pulmonaires à Pseudomonas aeruginosa}

Parmi les infections pulmonaires, celles dont sont victimes les patients atteints de la mucoviscidose sont difficiles à traiter à cause de leur caractère chronique dû à un terrain clinique favorable (réduction de l'hydratation des epithelia couplée à une hyperproduction de mucus). La bactérie que l'on retrouve le plus souvent chez ces patients est $P$. aeruginosa, bacille à Gram négatif naturellement résistant à plusieurs antibiotiques. De manière surprenante, il n'y a pas dans la littérature d'études menées chez l'animal pour tester le potentiel des bactériophages à traiter les infections pulmonaires causées par cet agent pathogène, à l'exception d'une expérience rapportée dans une publication plus large (Meitert et al., 1987). Par contre, il existe des bribes d'informations sur le traitement de patients. Une étude pionnière, réalisée en Union Soviétique en 1995, non disponible mais relatée par Sulakvelidze et ses collaborateurs, mentionne une efficacité des bactériophages dans le traitement de patients atteints de mucoviscidose infectés par P. aeruginosa (Sulakvelidze et al., 2001). Plus récemment, entre 2007 et 2010, une étude a été réalisée sur huit patients atteints de mucoviscidose à Tbilissi. Les patients ont reçu des bactériophages sous forme d'aérosols, à plusieurs reprises pendant 6 à 10 jours, avec maintien du traitement antibiotique conventionnel, des fluidifiants de mucus et des vitamines. Les résultats rapportés concluent à une diminution considérable de la concentration des bactéries dans les expectorations des patients, une amélioration de l'état de santé général et pour l'ensemble des patients cette phagothérapie a permis d'allonger les délais entre deux épisodes infectieux (Kutateladze \& Adamia, 2010). D'autre part, une étude de cas, également réalisée à Tbilissi, rapporte les mêmes conclusions pour un patient atteint de mucoviscidose souffrant d'infections chroniques à $S$. aureus et $P$. aeruginosa (Kvachadze et al., 2011). Ces études sont encourageantes et il semble que les bactériophages soient capables de réduire les infections à $P$. aeruginosa, cependant peu de patients ont été étudiés et il est donc difficile de conclure sur l'efficacité réelle des bactériophages, notamment sur le long terme. De plus, aucun détail sur les bactériophages utilisés, leurs natures, leurs doses ne sont disponibles.

Afin d'étudier plus précisément le potentiel curatif de bactériophages dans le traitement d'infections pulmonaires, nous avons isolé des eaux usées de la région parisienne plusieurs bactériophages capable d'infecter la souche PAK de P. aeruginosa. Cette souche provoque des infections létales chez la souris lorsqu'elle est inoculée par voie intranasale, mimant une voie d'infection naturelle. Une version modifiée de cette souche qui exprime les gènes luxABCDE, lui permettant de synthétiser de la lumière, a ensuite été utilisée pour suivre le développement in vivo de l'infection pulmonaire (figure 3). Les résultats obtenus avec ce système ont permis de montrer que les bactériophages 


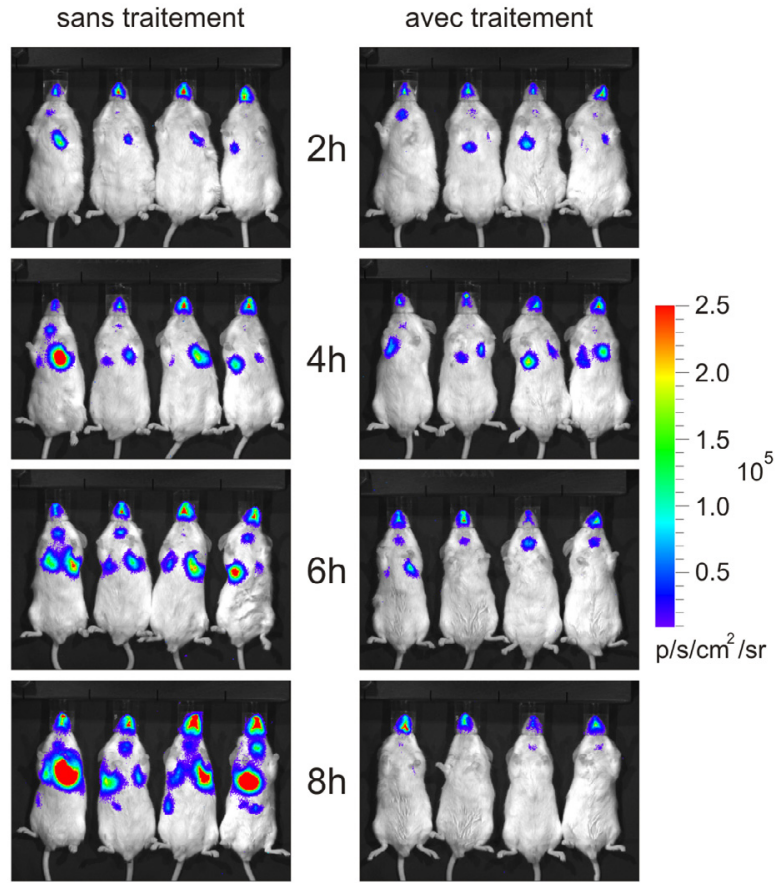

Fig. 3. Suivi de l'infection pulmonaire à $P$. aeruginosa (colonne de gauche) et de son traitement par un bactériophage (colonne de droite).

étaient rapidement actifs et permettaient la survie des animaux infectés par une dose létale (figure 3) (Debarbieux et al., 2010). Par la suite, un modèle similaire, basé sur l'utilisation d'une souche clinique de $P$. aeruginosa isolée d'un patient atteint de mucoviscidose, a permis d'étendre et de confirmer le potentiel curatif des bactériophages (Morello et al., 2011). Plus récemment, un autre groupe a publié des résultats similaires en utilisant d'autres souches de $P$. aeruginosa et d'autres bactériophages, élargissant encore un peu plus le potentiel de cette approche thérapeutique (Alemayehu et al., 2012). D'autre part, le potentiel préventif a lui aussi été mis en évidence. Chez la souris un prétraitement administré quatre jours avant une dose infectieuse létale permet la survie de plus de $90 \%$ des animaux. Ces résultats ont révélé une nouvelle facette de l'utilisation des bactériophages qui pourraient être utiles lors d'épidémies afin d'en limiter la propagation (Morello et al., 2011).

Le potentiel curatif des bactériophages pour traiter les infections pulmonaires ayant été démontré, comment peut-on envisager son application à l'Homme? L'innocuité des bactériophages est une question légitime bien qu'il soit difficile d'y répondre tant les données disponibles sur le sujet sont éparses. D'un côté, aucun effet négatif important n'a pu être associé à l'utilisation des bactériophages tant dans des modèles animaux que chez l'Homme dans les pays où cette approche est actuellement utilisée
(Pologne et Géorgie). Seule une élévation temporaire de la température a été remarquée de manière assez récurrente et qui est probablement consécutive à la destruction des bactéries qui s'accompagne de la libération d'un certain nombre de molécules ayant des propriétés immuno-stimulatrices. D'autre part, la réaction du système immunitaire vis-à-vis des bactériophages est un élément sur lequel très peu de données sont disponibles (Gorski et al., 2012). On considère donc que les bactériophages sont inoffensifs puisqu'ils ne sont pas capables d'infecter les cellules humaines, mais leur interaction avec le système immunitaire se doit d'être étudiée de manière plus systématique et approfondie (Miernikiewicz et al., 2013).

Toutes les infections pulmonaires aiguës ou chroniques sont-elles propices à un traitement par les bactériophages? Pour le cas des infections aiguës, qui sont des situations dans lesquelles les bactéries se développent rapidement, nous pouvons extrapoler les données obtenues chez l'animal et prévoir qu'un tel traitement sera efficace. Cependant, cette efficacité sera dépendante de la spécificité. Avant d'appliquer une solution de bactériophages, il sera nécessaire d'en vérifier l'activité sur les souches isolées des patients. Cette étape bien que rapide (moins de $24 \mathrm{~h}$ pour les bactéries à croissance rapide) est une étape essentielle si on veut garantir l'utilisation de la solution la plus appropriée. En ce qui concerne les infections chroniques, la situation peut paraître plus complexe mais elle est certainement moins urgente. D'une part, le terrain clinique est plus difficile, dans le cas de la mucoviscidose par exemple, avec la présence de mucus, de cellules inflammatoires, de protéases et autres facteurs qui peuvent perturber le bon déroulement de l'infection par les bactériophages. D'autre part, le fait de ne pas être placé dans une urgence permet de rechercher une optimisation de l'action des bactériophages en combinaison avec d'autres facteurs. On peut aussi prévoir que le traitement par les bactériophages doive être renouvelé à plusieurs reprises afin d'obtenir l'efficacité maximale, ce qui est à mettre en perspective avec l'éventuelle réponse du système immunitaire au cours de tels traitements. Enfin, un essai clinique de phase 2, effectué en Angleterre sur le traitement par des bactériophages d'otites chroniques à $P$. aeruginosa, a clairement illustré leur potentiel puisque tous les patients ont noté une amélioration de leur condition bien que la charge bactérienne n'ait pas toujours diminuée de manière conséquente (Wright et al., 2009).

\section{Infections intestinales à Escherichia coli}

E. coli est certainement la bactérie la plus connue par le grand public car elle fait régulièrement la une 
des journaux lors d'épisodes épidémiques causées par des souches pathogènes qui contaminent la chaîne alimentaire. En juin 2011, une épidémie touchant l'Allemagne et la France provoqua le décès de 53 personnes (Muniesa et al., 2012). La bactérie mise en cause était une souche de E. coli de sérotype O104:H4 présentant une très forte identité (plus de $99 \%$ ) avec une souche clinique dénommée 55989, qui fût isolée plusieurs années auparavant en Afrique chez un patient atteint de diarrhées persistantes. Ces souches appartiennent au groupe de $E$. coli entéro-agrégatives que notre laboratoire a choisi comme modèle d'étude des interactions entre bactériophages et bactéries dans l'intestin. En effet, la souche 55989 est capable de coloniser l'intestin de souris de manière prolongée sur plusieurs semaines sans causer de symptômes, nous offrant la possibilité d'étudier l'impact des bactériophages sur une longue durée. À cette fin, nous avons isolé trois bactériophages différents capables d'infecter cette souche. Leur étude a montré qu'ils s'agissaient de bactériophages virulents proches de bactériophages connus (Maura et al., 2012a). Seuls, mais surtout en cocktail, ces bactériophages sont tous capables d'infecter cette souche lorsqu'elle forme des biofilms in vitro. Administré pendant $24 \mathrm{~h}$ via l'eau de boisson à des souris non colonisées par la souche 55989 , le cocktail de ces trois bactériophages est rapidement éliminé (figure 4). Par contre, lorsque ce même cocktail est donné à des souris colonisées, nous avons observé que dans un premier temps la quantité de bactéries s'en trouve réduite (plus fortement dans l'intestin grêle) puis remonte à un niveau identique au groupe témoin des animaux non traités, et reste assez constant pendant au moins deux semaines (Maura et al., 2012b). Il est donc clairement démontré que les bactériophages sont capables d'infecter des bactéries présentes dans le tube digestif, provoquant une réduction du niveau de colonisation. D'autre part, le fait que l'intestin grêle soit plus affecté que le colon est probablement dû à une différence de l'état physiologique des bactéries qui sont alors plus ou moins sensibles à chacun des trois bactériophages. Nous avons en effet remarqué que si les trois bactériophages sont tous capables de se répliquer dans l'intestin grêle, seul l'un d'entre eux est capable d'en faire autant dans le gros intestin.

D'autres équipes se sont intéressées au potentiel thérapeutique des bactériophages ciblant des souches de $E$. coli et ont obtenu des résultats tantôt similaires tantôt différents (Tanji et al., 2005; Denou et al., 2009). Il en ressort que chaque bactériophage entretient avec son hôte une relation particulière qui est dépendante de l'organe dans lequel elle a lieu, cet organe étant lui-même soumis à la condition générale du patient. Par exemple, les diarrhées observées chez des patients peuvent certainement affecter les interactions
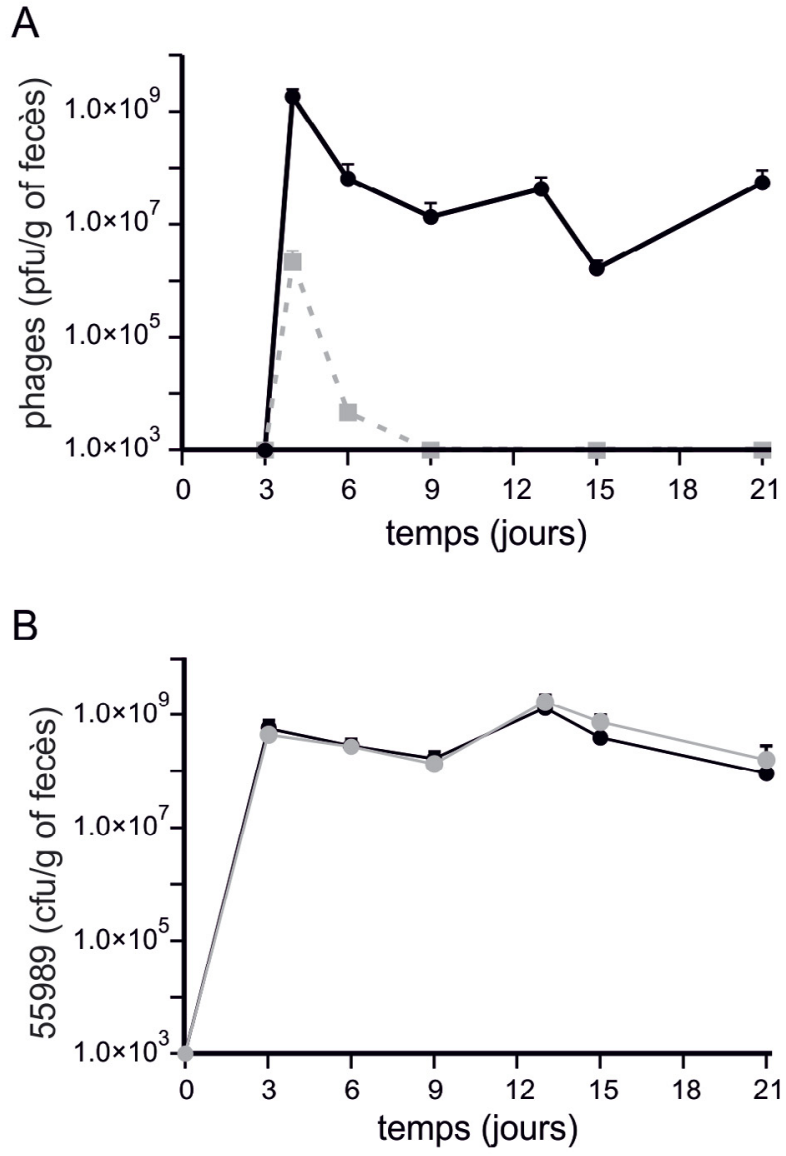

Fig. 4. Réplication in vivo des bactériophages dans l'intestin de souris. Quantités de bactériophages dans les fèces au cours du temps chez des souris non colonisées (carrés gris) et des souris colonisées par la souche 55989 (ronds noirs) (A). Quantités de la bactérie 55989 dans les fèces au cours du temps chez des souris colonisées par cette souche buvant pendant $24 \mathrm{~h}$ de l'eau de boisson ne contenant pas (ronds gris) ou contenant un cocktail de trois bactériophages (ronds noirs) (B), d'après (Maura et al., 2012a, 2012b).

entre bactériophages et bactéries. Ainsi, les relations entre E. coli et ses bactériophages (figure 5), que l'on pouvait considérer comme connues grâce aux études de biologie moléculaire, sont soudainement insuffisantes pour expliquer les observations obtenues à partir des modèles animaux. Dès lors que l'on introduit un partenaire supplémentaire, tout en se rapprochant des conditions environnementales naturelles pour E. coli, nous réalisons qu'une étude plus approfondie des relations hôtes-virus est nécessaire pour mieux comprendre les facteurs qui les influencent. Malgré plusieurs inconnues, le groupe Nestlé a récemment débuté une série d'études cliniques au Bangladesh pour évaluer l'innocuité d'un cocktail de bactériophages 


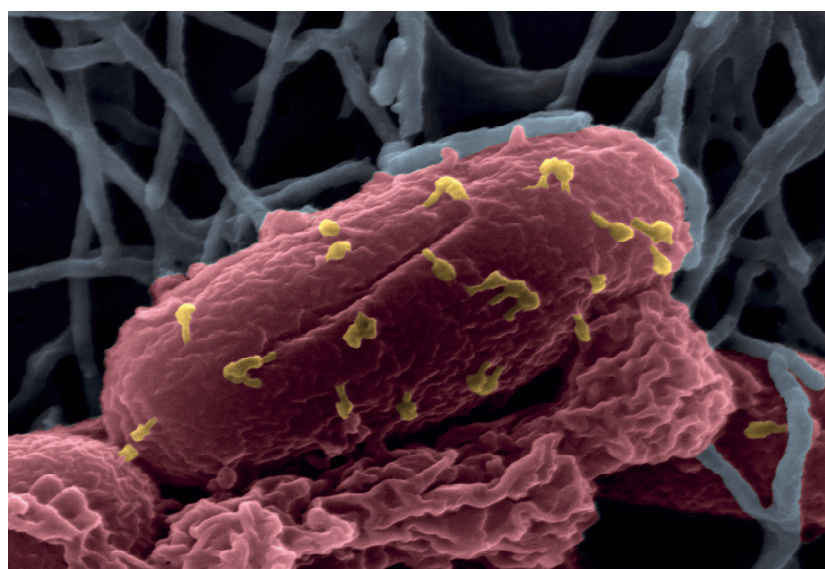

Fig. 5. Bactériophages (en jaune) à la surface d'une cellule de Escherichia coli (en rouge) (photographie prise en microscopie électronique à balayage puis colorisée).

dans le but de proposer une solution qui permettrait de réduire l'incidence des diarrhées infantiles causées par des souches pathogènes de E. coli (Sarker et al., 2012).

Les bactériophages sont donc des acteurs qui jouent un rôle dans le microbiote intestinal. L'importance de ce rôle reste encore à définir, suivant que l'on regarde l'homéostasie des populations bactériennes qui peuvent être soumises à des échanges génétiques via les prophages, ou la dynamique des populations qui peut être affectée par des bactériophages virulents. Que ce soit dans des pays possédant ou ne possédant pas un système de distribution et de traitements des eaux, l'Homme absorbe des bactériophages via l'eau potable (Lodder et al., 2010). À ce jour, aucune étude sur les conséquences de cette absorption ne semble avoir été menée, mais à la lecture des travaux étudiant la coévolution des bactériophages et des souches de Vibrio cholerae dans les eaux du Gange en Inde, le sentiment que les bactériophages pourraient participer à la guérison de certaines infections intestinales en sort renforcé (Faruque et al., 2005a, 2005b; Jensen et al., 2006). Enfin, les études de séquençage à haut débit des populations bactériennes et virales nous aideront certainement à mieux appréhender les mécanismes sousjacents aux interactions entre ces deux espèces dans l'intestin, dès lors qu'elles seront effectuées à des intervalles rapprochés de quelque jours et non pas de plusieurs mois.

Dans l'intestin, quels sont les éléments qui peuvent influencer l'utilisation des bactériophages dans une approche thérapeutique? En premier lieu, le fait que l'intestin abrite une communauté bactérienne complexe représente un challenge pour les bactériophages qui auraient pour mission de cibler spécifiquement une sous-population donnée. Ce défi repose sur la fréquence de rencontre entre les virus et leurs hôtes, sachant qu'elle n'est le fruit que du hasard, mais aussi sur l'accessibilité des souches ciblées, celles-ci pouvant être localisées à différents niveaux (lumière intestinale versus surface des cellules épithéliales). De plus, l'état physiologique des bactéries peut aussi être un facteur limitant, celui-ci pouvant rendre résistantes des bactéries sensibles. De plus, les bactériophages euxmêmes pourraient modifier leur comportement face à ces situations. Par exemple, opter pour un état de pseudolysogénie lorsqu'ils infectent un hôte qui ne présente pas les conditions physiologiques optimales pourrait leur permettre d'attendre des jours meilleurs une fois l'hôte placé dans un contexte plus favorable. Ne négligeons pas non plus le fait qu'à chaque multiplication virale, le génome des bactériophages va évoluer de manière très faible mais néanmoins suffisante pour générer des variants. Ceux-ci pourraient être plus compétitifs, voire devenir capables d'infecter des souches proches de celles initialement ciblées, et même éventuellement des souches commensales de E. coli par exemple. Tous ces évènements doivent certainement se combiner et il sera nécessaire d'en apprendre plus sur chacun d'entre eux pour arriver à favoriser ceux qui sont recherchés dans un but thérapeutique.

\section{Conclusions et perspectives}

Les résultats récents sont encourageants car ils démontrent clairement le bien-fondé d'une approche thérapeutique tombée dans l'oubli, mais qui finalement repose sur des interactions légitimes et naturelles entre des virus et leurs hôtes. Comment détourner et optimiser ces interactions pour les utiliser en médecine est le défi que nous devons relever afin de proposer une solution fiable pour le traitement des infections résistantes aux antibiotiques. Le chemin sera encore long car parallèlement aux différentes questions scientifiques évoquées plus haut, se posent des questions règlementaires (Brüssow, 2012). En effet, les bactériophages ne sont pas des médicaments classiques (il ne s'agit pas de molécules) et il n'existe pas de réglementation adéquate qui pourrait prendre en compte la nature particulière des bactériophages. À titre d'exemple, le devenir d'un médicament au cours du temps lorsqu'il est administré à un patient est connu : il est soit métabolisé, soit transformé, soit éliminé. Un bactériophage qui rencontre une population bactérienne, va disparaître pour produire de nouveaux bactériophages plus nombreux et dont la composition exacte sera légèrement différente. Ce sont des réalités que la règlementation doit accepter et intégrer pour proposer une solution adéquate qui permettra de développer la phagothérapie. 


\section{Références}

Alemayehu D., Casey P.G., McAuliffe O., Guinane C.M., Martin J.G., Shanahan F., Coffey A., Ross R.P., Hill C., Bacteriophages phiMR299-2 and phiNH-4 can eliminate Pseudomonas aeruginosa in the murine lung and on cystic fibrosis lung airway cells. M Bio, 2012, 3, e00029-00012.

Breitbart M., Rohwer F., Here a virus, there a virus, everywhere the same virus? Trends in Microbiology, 2005, 13, 278-284.

Brenner S., Jacob F., Meselson M., An unstable intermediate carrying information from genes to ribosomes for protein synthesis. Nature, 1961, 190, 576-581.

Brüssow H., What is needed for phage therapy to become a reality in Western medicine? Virology, 2012, 434, 138-142.

Clokie M.R.J., Millard A.D, Letarov A.V., Heaphy S., Phages in nature. Bacteriophage, 2011, 1, 31-45.

Crick F.H., Barnett L., Brenner S., Watts-Tobin R.J., General nature of the genetic code for proteins. Nature, 1961, 192, 1227-1232.

d'Herelle F., Essai de traitement de la peste bubonique par le bactériophage. Press Med, 1925, 1393-1394.

d'Herelle F., Le bactériophage et son comportement. 1926, Masson et Cie, Paris.

Da Costa Cruz J., Le traitement des dysenteries bacillaires par le bactériophage. CR Soc Biol, 1924, 91, 845.

Dang X., Yi H., Ham M.H., Qi J., Yun D.S., Ladewski R., Strano M.S., Hammond P.T., Belcher A.M., Virustemplated self-assembled single-walled carbon nanotubes for highly efficient electron collection in photovoltaic devices. Nat Nanotechnol, 2011, 6, 377-384.

Debarbieux L., Leduc D., Maura D., Morello E., Criscuolo A., Grossi O., Balloy V., Touqui L., Bacteriophages can treat and prevent Pseudomonas aeruginosa lung infections. J Infect Dis, 2010, 201, 1096-1104.

Denou E., Bruttin A., Barretto C., Ngom-Bru C., Brüssow H., Zuber S., T4 phages against Escherichia coli diarrhea: potential and problems. Virology, 2009, 388, 21-30.

Dublanchet A., Fruciano E., A short history of phage therapy. Méd Mal Infect, 2008, 38, 415-420.

Duerkop B.A., Clements C.V., Rollins D., Rodrigues J.L., Hooper L.V., A composite bacteriophage alters colonization by an intestinal commensal bacterium. Proc Natl Acad Sci USA, 2012, 109, 17621-17626.

Faruque S.M., Islam M.J., Ahmad Q.S., Faruque A.S., Sack D.A., Nair G.B., Mekalanos J.J., Self-limiting nature of seasonal cholera epidemics: Role of hostmediated amplification of phage. Proc Natl Acad Sci USA, 2005a, 102, 6119-6124.

Faruque S.M., Naser I.B., Islam M.J., Faruque A.S., Ghosh A.N., Nair G.B., Sack D.A., Mekalanos J.J., Seasonal epidemics of cholera inversely correlate with the prevalence of environmental cholera phages. Proc Natl Acad Sci USA, 2005b, 102, 1702-1707.

Gorski A., Miedzybrodzki R., Borysowski J., Da̧browska K., Wierzbicki P., Ohams M., Korczak-Kowalska G.,
Olszowska-Zaremba N., Łusiak-Szelachowska M., Kłak M., Joñczyk E., Kaniuga E., Goła A., Purchla S., Weber-Dąbrowska B., Letkiewicz S., Fortuna W., Szufnarowski K., Pawełczyk Z., Rogóż P., Kłosowska D., Phage as a modulator of immune responses: practical implications for phage therapy. Adv Virus Res, $2012,83,41-71$.

Gratia A., Studies on the D'Herelle Phenomenon. J Exp Med, 1921, 34, 115-126.

Gratia A., La lyse transmissible du staphylocoque. Sa production, ses applications thérapeutiques. CR Acad Biol, 1922, 86, 276-278.

Henry M., Debarbieux L., Tools from viruses: bacteriophage successes and beyond. Virology, 2012, 434, 151-161.

Jensen M.A., Faruque S.M., Mekalanos J.J., Levin B.R., Modeling the role of bacteriophage in the control of cholera outbreaks. Proc Natl Acad Sci USA, 2006, 103, 4652-4657.

Kutateladze M., Adamia R., Bacteriophages as potential new therapeutics to replace or supplement antibiotics. Trends Biotechnol, 2010, 28, 591-595.

Kvachadze L., Balarjishvili N., Meskhi T., Tevdoradze E., Skhirtladze N., Pataridze T., Adamia R., Topuria T., Kutter E., Rohde C., Kutateladze M., Evaluation of lytic activity of staphylococcal bacteriophage Sb-1 against freshly isolated clinical pathogens. Microb Biotechnol, 2011, 4, 643-650.

Labrie, S.J., Samson J.E., Moineau S., Bacteriophage resistance mechanisms. Nat Rev Microbiol, 2010, 8, $317-327$.

Lepage P., Colombet J., Marteau P., Sime-Ngando T., Doré J., Leclerc M., Dysbiosis in inflammatory bowel disease: a role for bacteriophages? Gut, 2008, 57, 424-425.

Lodder W.J., van den Berg H.H., Rutjes S.A., de Roda Husman A.M., Presence of enteric viruses in source waters for drinking water production in The Netherlands. Appl Environ Microbiol, 2010, 76, 5965-5971.

Luria S.E., Delbruck M., Mutations of bacteria from virus sensitivity to virus resistance. Genetics, 1943, 28, 491-511.

Luria S.E., Human M.L., A nonhereditary, host-induced variation of bacterial viruses. J Bacteriol, 1952, 64, 557-569.

Luria S.E., Delbruck M., Anderson T.F., Electron microscope studies of bacterial viruses. J Bacteriol, 1943, 46, $57-77$.

Maura D., Debarbieux L., Bacteriophages as twenty-first century antibacterial tools for food and medicine. Appl Microbiol Biotechnol, 2011, 90, 851-859.

Maura D., Galtier M., Le Bouguénec C., Debarbieux L., Virulent bacteriophages can target O104:H4 enteroaggregative Escherichia coli in the mouse intestine. Antimicrob Agents Chemother, 2012a, 56, 6235-6242.

Maura D., Morello E., du Merle L., Bomme P., Le Bouguénec C., Debarbieux L., Intestinal colonization by enteroaggregative Escherichia coli supports long-term bacteriophage replication in mice. Environ Microbiol, 2012b, 14, 1844-1854. 
Meitert E., Petrovici M., Sima F., Costache G., Savulian C., Investigation on the therapeutical efficiency of some adapted bacteriophages in experimental infection with Pseudomonas aeruginosa. Arch Roum Pathol Exp Microbiol, 1987, 46, 17-26.

Miedzybrodzki R., Borysowski J., Weber-Dąbrowska B., Fortuna W., Letkiewicz S., Szufnarowski K., Pawełczyk Z., Rogóż P., Kłak M., Wojtasik E., Górski A., Clinical aspects of phage therapy. Adv Virus Res, 2012, 83, 73-121.

Miernikiewicz P., Dạbrowska K., Piotrowicz A., Owczarek B., Wojas-Turek J., Kicieliñska J., Rossowska J., Pajtasz-Piasecka E., Hodyra K., Macegoniuk K., Rzewucka K., Kopciuch A., Majka T., Letarov A., Kulikov E., Maciejewski H., Górski A., T4 phage and its head surface proteins do not stimulate inflammatory mediator production. PLoS One, 2013, 8, e71036.

Minot S., Bryson A., Chehoud C., Wu G.D., Lewis J.D., Bushman F.D., Rapid evolution of the human gut virome. Proc Natl Acad Sci USA, 2013, 110, 12450-12455.

Modi S.R., Lee H.H., Spina C.S., Collins J.J., Antibiotic treatment expands the resistance reservoir and ecological network of the phage metagenome. Nature, 2013, 499, 219-222.

Morello E., Saussereau E., Maura D., Huerre M., Touqui L., Debarbieux L., Pulmonary bacteriophage therapy on Pseudomonas aeruginosa cystic fibrosis strains: first steps towards treatment and prevention. PLoS One, 2011, 6, e16963.

Muniesa M., Hammerl J.A., Hertwig S., Appel B., Brüssow H., Shiga toxin-producing Escherichia coli O104:H4: a new challenge for microbiology. Appl Environ Microbiol, 2012, 78, 4065-4073.

Prangishvili D., Forterre P., Garrett R.A.,Viruses of the Archaea: a unifying view. Nat Rev Microbiol, 2006, 4, 837-848.
Reyes A., Haynes M., Hanson N., Angly F.E., Heath A.C., Rohwer F., Gordon J.I., Viruses in the faecal microbiota of monozygotic twins and their mothers. Nature, 2010, 466, 334-338.

Sarker S.A., McCallin S., Barretto C., Berger B., Pittet A.C., Sultana S., Krause L., Huq S., Bibiloni R., Bruttin A., Reuteler G., Brüssow H., Oral T4-like phage cocktail application to healthy adult volunteers from Bangladesh. Virology, 2012, 434, 222-232.

Saussereau E., Debarbieux L., Bacteriophages in the experimental treatment of Pseudomonas aeruginosa infections in mice. Adv Virus Res, 2012, 83, 123-141.

Spellberg B., Powers J.H., Brass E.P., Miller L.G., Edwards Jr. J.E., Trends in antimicrobial drug development: implications for the future. Clin Infect Dis, 2004, 38, 1279-1286.

Sulakvelidze A., Alavidze Z., Morris Jr. J.G., Bacteriophage therapy. Antimicrob Agents Chemother, 2001, 45, 649-659.

Summers W.C., Félix d'Herelle and the origins of molecular biology. 1999, Y. U. Press, Yale.

Summers W.C., The strange history of phage therapy. Bacteriophage, 2012, 2, 130-133.

Tanji Y., Shimada T., Fukudomi H., Miyanaga K., Nakai Y., Unno H., Therapeutic use of phage cocktail for controlling Escherichia coli O157:H7 in gastrointestinal tract of mice. J Biosci Bioeng, 2005, 100, 280-287.

Taubes G., The bacteria fight back. Science, 2008, 321, 356-361.

Wommack K.E., Colwell R.R., Virioplankton: viruses in aquatic ecosystems. Microbiol Mol Biol Rev, 2000, 64, 69-114.

Wright A., Hawkins C.H., Anggård E.E., Harper D.R., A controlled clinical trial of a therapeutic bacteriophage preparation in chronic otitis due to antibiotic-resistant Pseudomonas aeruginosa; a preliminary report of efficacy. Clin Otolaryngol, 2009, 34, 349-357. 